\title{
Health Level Seven
}

National Cancer Institute

\section{Source}

National Cancer Institute. Health Level Seven. NCI Thesaurus. Code C80485.

A Standards Developing Organization (SDO) operating in the healthcare arena with an emphasis on the domain of clinical and administrative data. 